POS $\quad \begin{aligned} & \text { PROCEEDINGS } \\ & \text { OF SCIENCE }\end{aligned}$

\title{
WSRT Observations of Radio Source Structure in
} the 1970s

\section{George Miley}

Leiden Obs., Fac. Science, University Leiden, Postbus 9500, 2300 RA, Leiden, The Netherlands E-mail: miley@strw.leidenuniv.nl

50 Years Westerbork Radio Observatory, A Continuing Journey to Discoveries and Innovations Richard Strom, Arnold van Ardenne, Steve Torchinsky (eds) 


\section{Chapter 4 WSRT Observations of Radio Source Structure in the 1970 s}

George Miley*

$\mathrm{I}$ joined the Leiden-Westerbork group in December 1970, just as the WSRT was being commissioned. And the "Stichting" (forerunner to ASTRON) was still located in Leiden. My main scientific passion was and still is with the spectacular luminous radio sources that are associated with some galaxies and quasars. Having been "formed" scientifically" by a baptism in radio-link interferometry at Jodrell Bank and NRAO (hundred kilometre baselines and sub-arc second resolutions), I was sceptical as to whether the WSRT, with its low $\sim 20$ " resolution could contribute significantly to unravelling the nature of extragalactic radio sources. I was very wrong. The WSRT was indeed revolutionary. Its design was highly conservative one and in several ways copied the Cambridge One-Mile Telescope. However, with the large number of simultaneous baselines and collecting area, a redundant design and rock-stable electronics the WSRT probed a hitherto neglected parameter space: dynamic range - the ability to observe faint emission in the region of extremely bright radio sources. Its huge dynamic range enabled the WSRT to make many fundamental discoveries on the seventies and keep at the forefront of astronomy for the past 50 years.

Exploiting the unprecedented dynamic range of the WSRT was not straightforward. Until the early seventies radio source structure was displayed using contour maps, but these could not delineate the large contrast between high and low brightness structures revealed by the WSRT. Walter Jaffe, then a Leiden PhD student, tackled this problem by developing the then novel technique of displaying radio maps in the form of photographs. Walter's radiophotographs could show structures with an order of magnitude larger contrast than the old contour displays.

* Sterrewacht Leiden, Leiden University, The Netherlands
I will here mention three important discoveries that were made by simply inspecting images of radio source structure during the first decade of the WSRT (e.g. Miley, 1980, Ann. Rev. Astr. Astrophys. 18, 165-218):

1. One of the most intriguing characteristics of extragalactic radio sources is the narrow collimated double structure that emanate from the nuclei of their parent galaxies. The existence of giant radio sources with sizes spanning several megaparsec, such as 3 C 236 and DA 240 were first revealed 
by the WSRT (Willis, Strom \& Wilson, 1974, Nature, 250, 625). These large extended lobes had previously been missed by the Cambridge Telescopes due to their limited dynamic range and sensitivity.

A surprising related discovery was the demonstration the kiloparsec-scale radio nucleus of ${ }_{3} \mathrm{C}_{23} 6$ is aligned to within a few degrees with its megaparsec-scale structure. This meant that the collimation axis of 3 C236 must have stayed fixed for many tens of millions of years (Fomalont \& Miley, 1975, Nature, 257, 99). The long-term memory is presumed to be associated with the fixed rotation axis of the super massive black hole located deep in the nucleus of the radio galaxy.

2. "Radio jets", that are now known to channel the transport of energy from the galaxy nucleus out to the radio source lobes were first recognised and their name coined through WSRT observations of the radio source Bo844+31 (van Breugel and Miley, 1977, Nature, 265, 315). Subsequently M87-type optical emission was detected from WSRT radio jets in ${ }_{3} \mathrm{C}_{31}$ and ${ }_{3} \mathrm{C} 66$ using the video camera on the Kitt Peak $4 \mathrm{~m}$ telescope (Butcher et al. 1980, ApJ, $235,749)$.

3. One of the most spectacular early discoveries made by the WSRT concerned the nature of radio sources in clusters of galaxies. In the late sixties, the Cambridge group showed that two galaxies in the Perseus Cluster, NGC 1265 and IC 310 , had long radio tails that pointed away from the bright radio galaxy, NGC1275 at the centre of the cluster. They explained the strange narrow structures by a far-reaching hypothesis in which streams of electrons ejected from NGC 1275 travel for hundreds of kiloparsec and "light up" fainter radio sources in the other two galaxies (Ryle and Windram, 1968, MNRAS, 138, 1). Subsequently Hill and Longair found a comparable configuration around ${ }_{3} \mathrm{C}_{129}$ (Hill and Longair, 1971, MNRAS, 154, 125). They developed the galaxy interaction theory with a detailed model that explained how radio sources could be ignited over large distances, producing narrow radio tails that point away from the "igniter". Prompted by these results, we made deep WSRT observations of the same fields.

We were surprised to find faint a radio galaxy on the WSRT map of the Perseus Cluster that had a tail that pointed towards the presumed igniter, i.e. in the opposite direction to what was expected (Figure 1a). This was the nail in the coffin of the Cambridge interaction theory. But what could cause of the peculiar long narrow radio tails that were very different structures from "normal" double radio sources? The answer was clear from the high dynamic range from WSRT radiophotographs. These were developed by Herman Kleibrink, the head of the Leiden Observatory photographic department. "Mijnheer" Kleibrink was a brilliant photographer who had published several highly praised photo books of his beautiful Leiden. He was also a modest Dutch "gentleman" who wore a formal suit. When he showed me the radiophotograph of $3 \mathrm{C} 129$ he was excited. "Mr. Miley", he said. "this

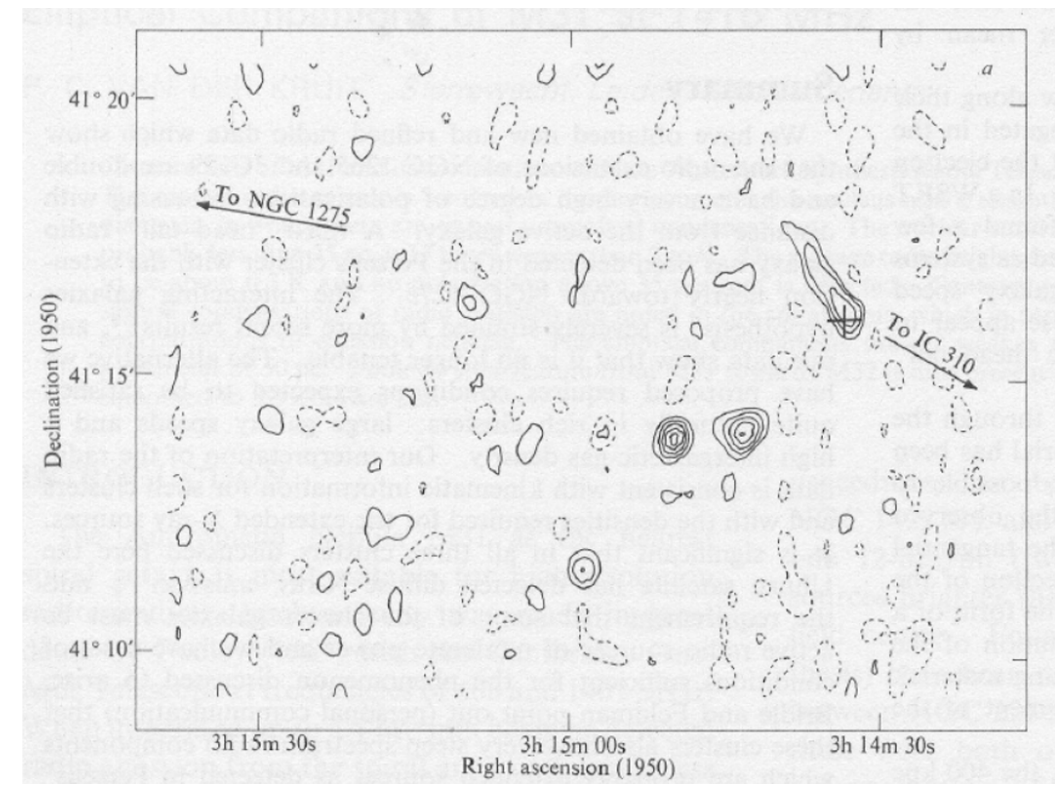
in Clusters.

1a. The presence of a hitherto unobserved weak tailed radio galaxy in a deep WhRT observation of the Perseus Cluster. in the "wrong" in the "wrong"

direction and was difficult to explain by the Cambridge of head tail radio of head tail radio

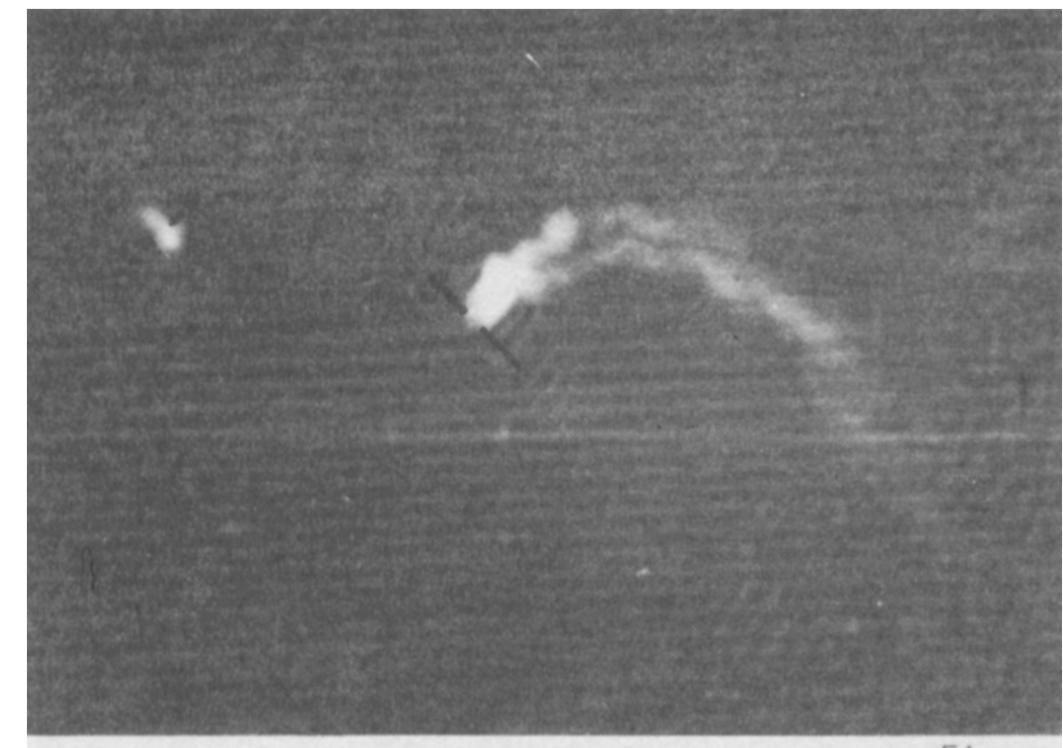

16. Herman Kleibrink's "World War $1 /$ fighter being shot down". Reproduction an original Jaffe WSRT radiophotograph of 3 C129 at $1415 \mathrm{MHz}$. It led to the idea that the narrow radio structures are trails left behind as the radio galaxies move through the cluster gas (Miley, Perola, van der Kruit and van der Laan, 1972,

3C129.1 and 3C129

looks just like a World War II fighters being shot down". Our subsequen paper indeed proposed that the radio tails are trails deposited by the host radio galaxies as they move through the cluster gas. (Figure 1, Miley, Perola, van der Kruit and van der Laan, 1972, Nature 237, 269). The WSRT discovery of radio trails began an important WSRT "industry" of cluster studies that involved e.g. Walter Jaffe, Cesare Perola, Alan Bridle, Galen Gisler and most prominently Ger De Bruyn. 
Figure 2. Sequence of radio-source bending, illustrated by schematic drawings of WSRT maps. This shows how the shape of radio sources in rich clusters can be influenced by relative motions of the host galaxies through the cluster gas. (Miley, 1980, Ann. Rev. Astron Astrophys. 18, 165-218).

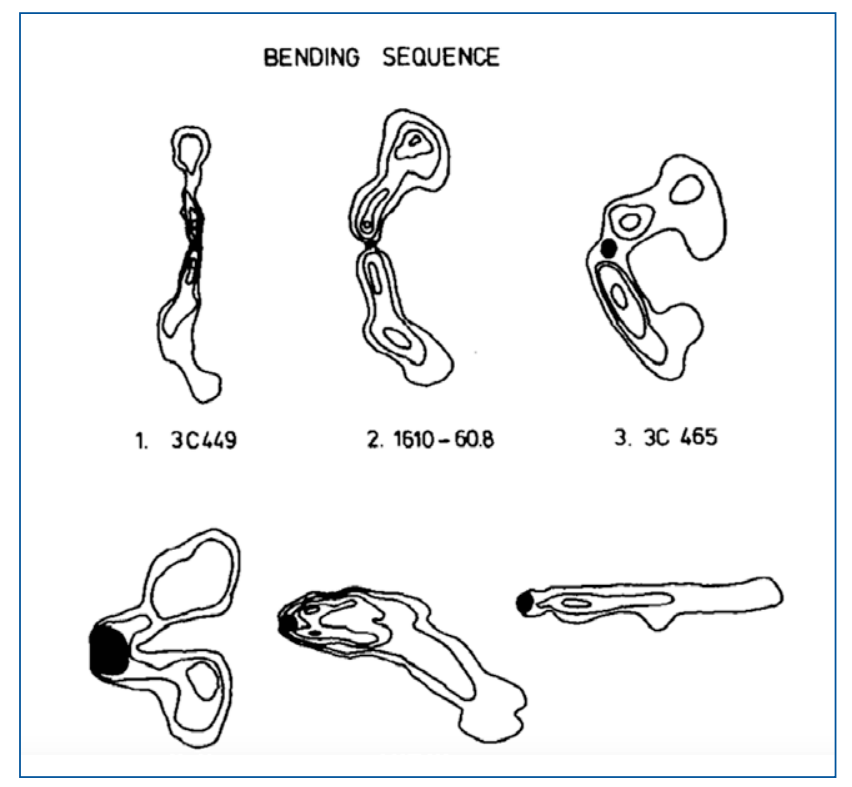

It was a great privilege to have had the opportunity of coming to Leiden and working with WSRT during the seventies. The youthful and exciting atmosphere provided by the gifted group of postdocs, $\mathrm{PhD}$ students and engineers under the leadership of Harry van der Laan and inspired by the enthusiasm and vision of Jan Oort was very special and made this era one of the most memorable in my life. I am delighted to congratulate the WSRT on being 50 years young. It's great to see that the "prima donna" is still very much alive and kicking and I'm sure that the renewed WSRT/Apertif will produce many exciting results in the future. 